\title{
Empathizing with patients: the role of interaction and narratives in providing better patient care
}

\author{
Carter Hardy ${ }^{1}$
}

Published online: 28 October 2016

(C) Springer Science+Business Media Dordrecht 2016

\begin{abstract}
Recent studies have revealed a drop in the ability of physicians to empathize with their patients. It is argued that empathy training needs to be provided to both medical students and physicians in order to improve patient care. While it may be true that empathy would lead to better patient care, it is important that the right theory of empathy is being encouraged. This paper examines and critiques the prominent explanation of empathy being used in medicine. Focusing on the component of empathy that allows us to understand others, it is argued that this understanding is accomplished through a simulation. However, simulation theory is not the best explanation of empathy for medicine, since it involves a limited perspective in which to understand the patient. In response to the limitations and objections to simulation theory, interaction theory is presented as a promising alternative. This theory explains the physicians understanding of patients from diverse backgrounds as an ability to learn and apply narratives. By explaining how we understand others, without limiting our ability to understand various others, interaction theory is more likely than simulation theory to provide better patient care, and therefore is a better theory of empathy for the medical field.
\end{abstract}

Carter Hardy

carterhardy@mail.usf.edu

1 Department of Philosophy, University of South Florida, 4202 E. Fowler Ave., FAO 280, Tampa, FL 33620, USA
Keywords Empathy · Education - Simulation theory · Interaction theory $\cdot$ Diversity problem $\cdot$ Narratives

There has been a lot of discussion in recent years concerning the role that empathy should play in medicine. After a long hiatus, the topic of empathy is now being revisited as something that is essential to good medical practice. According to a few recent studies, the physician's ability to empathize with patients is weakened during medical training, and this weakened ability can lead to a serious deficiency in patient care (Hojat et al. 2004, 2009; Eikeland et al. 2014; Suchman et al. 1997; Tavakol et al. 2012; Ward et al. 2012). In order to improve patient care, these studies also argue that empathy is a skill that should be fostered in a student's initial medical training. While any opportunity to improve patient care should be supported, and the human ability to empathize does have its merits in regard to our care for one another, proponents of empathy need to be careful.

The term "empathy" is polysemic, making it difficult to argue that there is one correct definition. ${ }^{1}$ The discussion of empathy in different fields of research has attracted a large number of different definitions and explanations (Batson 2009; Gelhaus 2012a, p. 106; Lanzoni 2012; Pedersen 2008, p. 327). Various responses have been defined as empathy_including what might

\footnotetext{
1 The term "empathy" has a rich history in both philosophy and aesthetics, and the translations of other terms such as the German terms Einfühlung and Mitgefühl to the English term empathy often seem arbitrary (Lanzoni 2012, p. 306). As such, it is important to make sure that a terminological debate is being avoided. It is important that empathy is being discussed based on how well the phenomenon attached to the term is being explained.
} 
also be called sympathy and emotional contagion-and then different explanations given for even the same definition. As such, the term tends to adopt a meaning that is the most pragmatic for a field. However, this does not mean that the adopted explanation for empathy is in fact the best explanation for that field. We still need to assess whether or not the right definition for empathy is the one that is being applied. If the wrong kind of ability is attached to the term, then we risk encouraging the wrong kind of behavior in physicians.

In order to best assess whether the definition being used for medical empathy is in fact the best definition for medical practice, I address the topic in three parts. First, I explain the likely causes for the lack of empathy in medicine and why this is problematic for both patients and physicians, focusing specifically on the scientific attitude that underlies these causes. Second, I examine the simulation theory of empathy (henceforth ST), which is one possible and popular theory of empathy that has appeared promising for teaching physicians to empathize. Empathy achieves an understanding of the other through a simulation of the other's mental states. In this section, I also explain the theoretical and ethical problems that arise for ST, making it a poor fit for medical empathy. Since ST limits the ways in which we can understand others, by restricting us to what we can either simulate or imagine about the other's situation, it alone does not match the actual kind of experience we want in a physician. In other words, ST does not provide the physician with the understanding of the patient that empathy is supposed to provide. Finally, I present Shaun Gallagher's interaction theory (hencefort IT) as a promising alternative explanation for medical empathy. IT explains how we can understand others from various backgrounds in a wide variety of situations. Additionally, the need to interact with patients helps to encourage open communication and it empowers patients by allowing them to be an active part in their treatment.

\section{A problematic lack of empathy}

\section{The negative view of empathy in medicine}

Many have noted and discussed the difficulty of current medical students and professionals to empathize with patients (Eikeland et al. 2014; Hojat et al. 2004, 2009; Suchman et al. 1997; Tavakol et al. 2012; Ward et al. 2012). Studies have made use of multiple tools to measure this decline in empathy, including the Jefferson Scale of Physician Empathy (henceforth JSPE), the Interpersonal Reactivity Index, and questionnaires (Hojat et al. 2004, pp. 935-936, 2009). ${ }^{2,3}$ In studies by Eikeland et al. and Hojat et al., it was argued that medical students are specifically trained to lack empathy; not explicitly in their curriculum, but rather as a side effect of the attitude required to get through medical school (Eikeland et al. 2014; Hojat et al. 2009). This drop in empathy appears to have something to do with the education of medical students, occurring around the third year of medical training (Eikeland et al. 2014; Hojat et al. 2009). ${ }^{4}$

A number of features of medical education have been credited with deadening a student's ability to empathize. Among these are the limited amount of time for students to learn copious amounts of information (Eikeland et al. 2014, p. 4; Hojat et al. 2009, p. 1188), the belief that emotions distract physicians from making good decisions (Eikeland et al. 2014, p. 4), and the development of cynicism as a necessary coping technique to avoid attachment and professional burnout (Eikeland et al. 2014, p. 5; Halpern 2011; Hooker 2015, p. 542; Testerman et al. 1996). ${ }^{5}$ As a result of these different features, empathy is not only set aside in favor of more pressing concerns; it actively trained away. It is understood as something extraneous and dangerous for physicians.

However, the blame should not be entirely placed on the intensity of medical education. Others have also noted that empathy is weakened in medical students due to the lack of role models who exemplify the positive role of empathy in medicine (Eikeland et al. 2014; Marcus 1999; Skeff and Mutha 1998). There is already an established attitude in the

\footnotetext{
$\overline{2}$ The JSPE is particularly important because it was specifically designed to measure empathy in medical practitioners. While there are other measures for empathy, this was the first developed specifically for the medical field (Hojat et al. 2004, pp. 935-936, 2009, p. 1183). The JSPE has two forms: one for measuring the ability to empathize in medical students (S-Version) and the other to measure the same ability is physicians (HP-Version) (Hojat et al. 2004, p. 936, 2009, pp. 1183-1184). In order to develop the JSPE, researchers first needed to settle on a specific definition for empathy, as well as an explanation of how we empathize (Hojat et al. 2004, p. 936). It is only with this in place that researchers were able to design a scale to measure empathy as it is defined. This scale was then tested and refined based on how well it actually measured empathy. The final result is a list of 20 items that medical students and physicians must answer. The answers to the questions are provided on a 7-point Likert scale, based on how strongly they either agree or disagree with the given statements (Hojat et al. 2004, p. 936, 2009, p. 1183).

3 The JSPE is often criticized as discussing empathy too metaphorically as "levels" (Hooker 2015, p. 543), as well as for only really measuring the medical student's and/or professional's belief in the importance that empathy serves for medicine (Pedersen 2009). That is, they do not actually measure empathy itself but rather one's belief that empathy is either important or unimportant.

${ }^{4}$ By testing medical students with the student version of the JSPE at both the beginning and the end of their third year, it was determined that this is when empathy seems to be the most affected (Hojat et al. 2004, p. 937).

5 Halpern call this "compassion fatigue" (Halpern 2014, p. 301).
} 
medical field that condemns empathy and encourages scientific objectivism. Traditional medicine is characterized by a drive to give objective advice to the patient, rather than to connect with the patient (Eikeland et al. 2014, p. 4; Singh 2005; Khanuja et al. 2011, p. 37; Pedersen 2010, pp. 598-599). As such, the suppression of empathy is seen as a desirable skill for a physician, rather than as a problem to be solved. Physicians embody this scientific attitude, and students emulate these physicians. By following in these footsteps, students train themselves to have different dispositions-ones that do not cause them to react empathetically to their patients-entrapping medical students and physicians alike in a vicious circle. This points to the scientific attitude - as it has been adapted for medicine - as being an underlying cause of the lack of empathy (Eikeland et al. 2014, p. 3; Gelhaus 2012a; Halpern 2001, 2011; Hojat et al. 2009, pp. 1188-1189). ${ }^{6}$

By scientific attitude, I mean that specific attitude noted above that focuses on objectivity and detachment in order to best understand and judge a situation. This attitude has undoubtedly been advantageous for medical science, aiding in the production of progressively improved technology and treatments. However, when applied to medical practice, this attitude may be detrimental to good patient care. As it has been adapted for medicine, this equates to making medicine as much like science and physicians as much like scientists as possible. It is this attitude that is exemplified in the structure of medical education and the physicians that serve as role models for students. Assuming that there is an objective answer to every condition, students focus all of their efforts into accumulating information, often to the detriment of their affective lives. ${ }^{7}$

The fear here is that affective practices like empathy will lead to a close connection between patient and physician, and that this connection to patients would cause emotional fatigue and professional burnout (Hooker 2015, pp. 543-544). Additionally, if physicians become too

\footnotetext{
${ }^{6}$ To be fair, it should be noted that "empathy did not decline for some students (a minority of 27\%) [which] suggests that there may be certain protective factors that defuse the harmful influences" (Hojat et al. 2009, p. 1189). This could mean that some students did not adapt this attitude towards medicine and therefore continued to believe that empathy is important.

7 There is an additional background assumption here that affective practices cannot provide objective knowledge. They only allow for subjective understanding of oneself. As such, affects are traditionally seen as contrary to reason, and therefore will more often than not either mislead a physician's rational judgments or provide false information themselves (Eikeland et al. 2014, p. 4; Halpern 2001, p. 30). Though this view has not been entirely rejected, it is fairly outdated. Most philosophers accept that at least some affects, such as emotions, have rational components. Emotions do not act contrary to reason. On the contrary, they are essential to reason, either by acting as judgments of the world or by focusing our attention such that we can make judgments.
}

emotionally invested in the patient's situation, they are more likely to have poorer judgment with regard to treatment than if the physician maintained an emotional distance (Eikeland et al. 2014, pp. 3-4; Hooker 2015, p. 544). Therefore, if empathy is avoided, then physicians will be able to maintain a professional distance from the patient. The less of an emotional connection the physician shares with the patient, the easier it will be for the physician to approach and solve the patient's problems objectively. Essentially, students held the belief that physicians are supposed to be like scientists. They should be unbiased and capable of disinterested observation, which should lead to an objective answer concerning the patient's problems. As such, rather than feeling with the patient, medical students become cynical observers as a desired coping strategy (Eikeland et al. 2014, p. 5). By distancing themselves from patients, the students are not absorbed into the patients' lives and pains, and therefore are less burdened by the ways in which their decisions affect patients.

\section{The benefits of empathy for patients and physicians}

It has become more common in recent years to reject all of these negative views of empathy, and to see the benefits that empathy can have in the medical field. There has been a turn away from the characterization of a physician as being a cold, detached observer of the facts. The scientific attitude is taken to be important and useful, but not the only relevant skill required for medicine (Gelhaus 2012a, p. 104). The benefits of empathy, sympathy, and care are gaining increasingly more attention, and arguments are being made to revalue them in medicine.

It has been shown that physicians who can empathize with their patients provide better care than those who are unable to empathize (Eikeland et al. 2014; Derksen et al. 2013; Di Blasi and Kleijnen 2003; Halpern 2001; Hojat et al. 2011, 2013; Hooker 2015; Pedersen 2008; Roter et al. 1998). Empathy greatly enhances the physician-patient interaction, allowing for the physician to better understand the patient's experiences, and therefore better understand how the patient should be treated. Much of the information given by a patient is hinted at nonverbally, meaning that physicians need to be able to understand these hints and know when to ask for more information (Finset and Mjaaland 2009; Halpern 2014; Suchman et al. 1997). Doing so leads to patients sharing more information about their situations than they might have shared otherwise. That is, empathy leads to more information being given concerning the situation of the patient, which also makes it more likely that the physician will be able to "correctly diagnose and effectively treat medical problems" (Halpern 2014 , p. 303). Patients are more likely to trust empathic physicians and better understand the treatment options 
being offered because the patient perceives the physician as genuinely caring about the patient's situation (Halpern 2001, 2014; Kim et al. 2004; Roter et al. 1998). Additionally, empathy decreases patient anxiety and allows them to feel empowered (Derksen et al. 2013; Halpern 2014 , p. 303). Patients feel like they are part of the medical encounter, that their input matters, and that they have a say in what will happen to them.

It is also argued that empathy is beneficial for the health of the physician (Gleichgerrcht and Decety 2013; Eikeland et al. 2014; Halpern 2014; Larson and Yao 2005; Roter et al. 1998). Among these benefits are "increased diagnostic accuracy, more meaningful work, an increased sense of well-being, and reduced symptoms of burnout" (Eikeland et al. 2014, p. 1). Additionally, physicians who experience empathy for their patients feel more fulfilled by their work than those who are unable to empathize (Halpern 2014, p. 304; Larson and Yao 2005, Shanafelt et al. 2005). This adds to the overall well-being of the physician and challenges the previous assumption that empathy is dangerous for the physician's mental health. While the connections made through empathy do bring the risk of emotional attachment, empathy also allows physicians to value their work more while experiencing less fatigue.

If it is true that empathy will lead to better patient care and physician well-being, then it makes sense to argue that steps need to be taken to revalue empathy in medicine. It is strongly argued that the attitudes and practices that deaden empathy need to be removed, and that empathy needs to be retaught to those who have lost it (Hojat et al. 2009; Khanuja et al. 2011; Pedersen 2010; Singh 2005; Williams et al. 2015). Empathy training needs to be introduced as part of the curriculum so that it is perceived by students as a useful skill to be fostered, rather than an extraneous skill that can be neglected. Additionally, there needs to be a change in attitude for their physician role models. If their role models lack empathy, then students won't see empathy as an important skill. Therefore, physicians should also receive empathy training. This will serve to improve their own skills, as well as allow them to be positive role models for future physicians. However, even if it is accepted that empathy is both lacking and needed in medical practice, it still needs to be questioned what kind of empathy ought to be encouraged to bring about the desired benefits.

\section{The simulation theory of empathy}

\section{Simulation theory for medical practice}

The previous section discussed the benefits of empathy, but this is slightly misleading, since the different individuals explaining these benefits have different opinions of how empathy should be understood. As noted earlier, empathy is polysemic. Some discuss empathy as an isolated experience, others as a group of different experiences that can be applied in different situations (Halpern 2014, p. 304), and still others who think empathy alone is not enough, and must be supplemented with other experiences like sympathy and care (De Vignemont and Jacob 2012; Gelhaus 2012a, b, 2013; Svenaeus 2015).

Due to the many different ways empathy can be defined and the different experiences that can be perceived as important for empathy, it would be too large of a task in this paper to assess every possible definition and explanation. Therefore, I limit the scope of this paper to the different explanations given for one of the most essential components of a definition of empathy: that it is an understanding of the other subject. This is one of the most common components used to define empathy in medicine (Pedersen 2008, p. 325), and in the context of medicine, this would mean that empathizing with a patient should allow physicians to better understand the patient's thoughts and feelings. However, there are still different theories concerning how this understanding is achieved.

As this aspect of empathy is most commonly discussed in the medical field, it is an understanding of the other that is achieved through a kind of simulation of the other's mental states (Cohen et al. 2011, p. 1639; Eikeland et al. 2014, p. 1; Hojat et al. 2001, 2004, p. 935, 2009, p. 1183; Khanuja et al. 2011, p. 37). ${ }^{8}$ In other words, empathy is understood as the physician's ability to place himself or herself into another's shoes (Pedersen 2010, p. 600; Williams et al. 2015, p. 1; Hojat et al. 2009, p. 1184). Defined as such, this theory of empathy corresponds to the simulation theory of empathy (ST). According to ST, we understand the mental states of others by simulating them. It is our ability to see the world from the other's point of view, thereby gaining an understanding of the other's experiences of the world (Gladstein 1983, p. 472; Rushton 1980, p. 37). When we observe the other acting in some way, we simulate the actions within ourselves, as if we had the other's perspective, reflect on the mental states that arise within us, and then project these mental states into the other. This would mean that physicians understand their patients in so far as they are able to mimic the mental states of their patients, either consciously or subconsciously. If the patient is feeling a pain in his or her arm, physicians imagine what it would be like if they had a similar pain, then apply to the patient the sensations, fears, desires, and

\footnotetext{
8 The belief that empathy is a simulation of the other is not new. ST has a rich tradition dating to Theodore Lipps, who adapted a theory of aesthetic empathy to be used in philosophy of mind (Lanzoni 2012, p. 306).
} 
so on that arise with that pain. Physicians understand patients because they can be in a state like their patients.

\section{Objections and limitations}

If empathy is meant to improve patient care due to the way that it provides us with an understanding of the other, then we need to assess whether or not a simulation actually provides physicians with an understanding of their patients. While this may initially seem like a promising way to explain empathy, it can easily be demonstrated that there is, as Edith Stein says, an inconsistency between "the phenomenon to be explained and that actually explained" (Stein 1989, p. 23). ${ }^{9}$ In other words, while the initial goal is to explain how we understand others, this is not actually explained by a simulation of the other.

The physician's understanding of the patient is entirely dependent on what the physician can understand about himself or herself. By simulating the patient's mental state, the physician gains an understanding of what it might be like for the physician to be in the patient's shoes. However, this does not imply that the physician actually knows what it is like to be the patient in the patient's shoes. In other words, when the physician simulates the patient, the physician's understanding is limited to what the physician can imagine about the patient's experiences. This objection is what some philosophers have called the diversity problem (Gallagher 2012, p. 363). That is, if all I can understand of the other when I empathize with the other is what I can simulate within myself, then my understanding of diverse experiences is limited to what I have-or could have-experienced. Therefore, under this argument, there is no diversity in the world for the physician. There is only the spectrum of a single self: the empathizing physician.

As Gallagher notes, there may be ways for ST to address the diversity problem (Gallagher 2012, p. 370). ST may want to argue that my understanding of the other simply is limited to what I can understand based on my own experiences. That is, the need to use my understanding of myself in order to understand others is simply an unavoidable restriction of my ability to understand others. It is something that we must acknowledge and live with (Gallagher 2012, p. 370). However, this is a questionable conclusion. As Gallagher says, it is possible in at least some cases to "empathize with monsters or aliens from other planets, as portrayed in film, and we can empathize with humans who live in far away lands and who are very different" (Gallagher 2012, p. 370). This, however, relies on us having good knowledge of their narrative, which will be explained further in the next section. ST, on the other

\footnotetext{
9 Stein has her own arguments against simulation-based theories of empathy. They can be found in her work On the Problem of Empathy.
}

hand, would have to argue that this understanding of very different others is not possible, since we cannot actually simulate the perspectives of monsters and aliens that are very different from us.

The diversity problem is particularly problematic in medicine, since it implies that the patient's experiences are always understood from the physician's perspective rather than that of the patient. As Claire Hooker notes, the physician's simulated understandings of the patient "mostly turn out to be merely chimeric projections" (Hooker 2015, p. 542). They ignore the fundamental differences between the physician and the patient, as well as the differences between individual patients. Additional problems arise if the physician and patient are of different genders, races, cultures, economic classes, and so on. Especially since several of these problematic factors are not directly observable, it is risky to assume that the physician can actually understand the patient via a simulation. If physicians are being affected by the situation and the other, but make a decision without being aware of their own emotional states-thinking that they are being objective-then they "risk making poor decisions to alleviate their own distress" (Halpern 2014, pp. 305-306).

A related problem is what some philosophers call the geocentric bias (Zahavi 2014, pp. 106-107). Essentially, ST also risks applying too much of our own situation to the other. It assumes that the other has knowledge, experiences, and skills like the subject, even when the other is not warranted to have these. For instance, if the physician simulates the other, it is difficult for the physician to forget all of his or her own background knowledge learned during medical training. However, it would be dangerously inaccurate for any of this knowledge to slip into the physician's simulation. This would grant the patient far too much credit, especially when it is often the case that patients are very ignorant of relevant symptoms and possible treatments. An important aspect of the physician-patient interaction is a respect for different positions and experiences, but this is not accomplished in simulations.

While the diversity problem and the geocentric bias are both problematic for ST, there is another problem with ST that ties directly to traditional medicine as it was addressed earlier. That is, even though ST is a theory of empathy, it is one that does not escape the scientific attitude. Physicians try to understand their patients by placing themselves in their patients' shoes, but this is still an approach to the other as completely separate from oneself and only capable of being understood from this perspective. In this way, ST takes a scientific attitude towards understanding the other. This makes it easy to incorporate ST into medicine, but it is one of the causes for the lack of empathy in the first place. Trying to adopt a theory of empathy that fits this attitude perpetuates the problem rather than solves it. In other 
words, rather than escaping the scientific attitude, simulation theory appropriates empathy into a purely cognitive skill that fits with the scientific attitude (Garden 2007; Halpern 2003; Macnaughton 2009; Pedersen 2008). ${ }^{10}$ Medicine has defined empathy in a way that is useful to its current attitude, maintaining the overall authority of the medical practitioner. As a result, the physician risks taking a paternalistic position to help the patient.

To summarize, ST has two main problems for medical empathy. First, it does not go far enough in its explanation of how we understand others. As evidenced by the diversity problem and the geocentric bias, simulations do not actually provide an understanding of the other. The part that ST does explain - the very basic understanding of another's mental state-is far too common and immediate to suppose the need of a complex process like simulation. As Halpern says, "it is often quite obvious that a patient is sad versus angry, and the crucial aim is to learn what in particular is bothering this individual" (Halpern 2014, p. 303). This latter part is not achieved by a simulation, so a different theory of empathy is needed to reach this goal. Second, ST does not escape from the scientific attitude, but is rather appropriated by medicine to fit with this attitude. Adopted in this way, empathy perpetuates the problems listed in the first section rather than solves them. In other words, if the purpose of empathy was to reconnect us to others and allow us to overcome the purely scientific attitude, then ST fails to be the theory of empathy that is needed. The patient is still being treated as an object for observation and judgment, rather than a subject with whom physicians interact. Therefore, we need a different explanation of how empathy allows us to understand others-one that will best fit medicine and aid in producing better patient care.

\section{Interaction and narrative}

\section{Interaction theory}

One promising alternative to the ST approach is Gallagher's interaction theory (IT). This theory seeks to draw on insights from phenomenology in order to provide a better explanation of how we understand others. IT consists of three levels: primary intersubjectivity, secondary intersubjectivity, and narrative competency (Gallagher 2009,

\footnotetext{
$\overline{10}$ One of the ways that this is done is by defining the cognitive skill of understanding others as empathy and the affective skill of feeling for others as sympathy (Gerdes 2011; Hojat et al. 2009). Following this, it is not uncommon to then praise empathy and condemn sympathy as the ability that physicians are actually trying to avoid (Hojat et al. 2009, p. 1183; Svenaeus 2015, p. 275). In this way, all of the negative effects that were traditionally associated with empathy are now associated with some other phenomenon.
}

p. 292). The first and the second levels explain the ways in which we immediately understand others as being with us in the world (Gallagher 2004, p. 204). The third level concerns our ability to understand particular others from diverse backgrounds. As such, it is the third level that is the most relevant for the current discussion. Since primary and secondary intersubjectivity are foundational for our everyday social interactions, it is unlikely that they can be easily lost or gained. It would likely take some kind of physical or psychological trauma for them to be lost. Narrative competency, on the other hand, can be lost or gained. Depending on our effort to learn other narratives, we can either possess many, diverse narratives or few, similar narratives. Additionally, we can be better or worse at constructing narratives with others. Therefore, if there is a concern that physicians lack empathy, then narrative competency would be the skill that needs to be retrained. However, narrative competency is based on a foundation established by the previous two levels, so they must be explained first in order to demonstrate the overall benefits of IT.

Primary intersubjectivity "includes some basic sensorymotor capacities that motivate a complex interaction between the child and others" (Gallagher 2009, p. 292). It develops very shortly after birth, as evidence by the phenomenon of neonate imitation (Gallagher 2004, p. 205, 2009, pp. 292-293; Gallagher and Meltzoff 1996; Meltzoff and Moore 1977). Even a few hours after birth, infants are capable of mimicking the gestures and expressions of others. This is an impressive skill that demonstrates the infant's very basic understanding of others. The infant can see the other's expression, somehow know that its face is similar, and then move its face to match. No simulation is needed in order for the infant to understand the other, since infants are incapable of running simulations (Gallagher 2004, p. 206). ${ }^{11}$ They cannot abstract themselves from interactions with others in the way that adults can (Gallagher 2009 , p. 293). Infants initially come to understand others because they are enmeshed in interactions with others, not because they can observe and simulate others.

Secondary intersubjectivity (which develops around 1 year of age) is based on "the development of joint attention, and motivates contextual engagement, and acting with others" (Gallagher 2009, p. 292). At this level, we start to understand others in the context of a world, and the world begins to take some of the focus of our interactions with others (Gallagher 2009, p. 294). Social situations, environment, and the objects around infants create a context in which they can understand others in a specific way (Gallagher 2004, p. 207, 2009, p. 294). They pay attention

\footnotetext{
$\overline{11}$ ST may be able to provide an answer to this problem by appealing to mirror neurons, the possibility of which is addressed below.
} 
to and make reference to the things around them, such that the environment is an essential part of how they understand others. The intentions of another person's gestures as they relate to the local environment and their intentional objects in the local environment come together in our understanding.

Narrative competency "involves narrative practices that capture intersubjective interactions, motives, and reasons" (Gallagher 2009, p. 292). Built on secondary intersubjectivity, language acquisition, and the stories that we tell children, narratives expand on the already established understanding of others, and start to "provide more subtle and sophisticated ways of framing the meaning of the other's intentions and actions" (Gallagher 2009, p. 294). Narratives allow us to interact with others in more complex ways, allowing both oneself and others to make sense of the world with joint-narratives (Gallagher 2012, pp. 369-370). We then use these complex, communally established narratives to understand the actions and intentions of both others and ourselves (Gallagher 2009, p. 294).

Early in childhood (around 2-4 years of age) children tend to adopt the stories of others, and later (around 4 years of age) they begin to contrast others' stories with their own experiences to create more diverse narratives (Gallagher 2012 , p. 370). Using merely my own understanding of myself as a means of understanding others, it can always be asked whether I actually understand the other, or I really only understand myself (Gallagher 2012, p. 370, 373). Narratives on the other hand are not limited to my narrow perspective. I have the narratives that I created as well as those learned from others. Through my interactions with others, these narratives and the ways that they relate to one another intertwine, refining my narratives to apply to increasingly more complex situations. These refined narratives become essential for explaining the reasons behind actions and behavior (Gallagher 2012, p. 371).

For physicians, primary intersubjectivity, secondary intersubjectivity, and narratives provide a structure and a context in which they can understand patients (Gallagher 2012, p. 371). Primary and secondary intersubjectivitywhich are still present and important even after the acquisition of narrative competency (Gallagher 2009, p. 293) - provide the physician with a very basic understanding of patients in their everyday interactions with their patients. Without this basic understanding, interactions with patients would not even be possible. Physicians can then use narratives to make sense of their patients in many different situations. Since patients come from various backgrounds and can be afflicted by various problems, physicians need a wide variety of narratives in order to best understand their patients. They must also be able to refine these narratives with their patients in order to best understand the patient's situation. With these narratives, physicians are able to overcome problems that $\mathrm{ST}$ is not able to overcome.

\section{The benefits of interaction theory}

To begin with, IT overcomes what Gallagher calls the "starting problem" (Gallagher 2012, pp. 371-372). For ST, we understand others by simulating their mental states within ourselves, but it is unclear how we know what to simulate before we understand the others' mental states. How does the understanding of the other-the simulation-start if we do not already understand the other? This is not a problem for IT, since it argues that "I draw on a rich store of narratives, and on the massive hermeneutical background that informs my understanding" (Gallagher 2012, p. 372). In other words, when physicians are interacting with patients, they can gain an understanding of their patients' mental states by situating them in the narratives, which the physicians have acquired throughout their lives. These narratives tell them what to expect from patients given their actions and situation (Gallagher 2012, p. 371). Physicians do not need simulations to create some new understanding; they already possess a wide variety of narratives that can be applied based on the patient's actions. When physicians interact with patients, they already have a basic understanding of them based on primary and secondary intersubjectivity. Based on this immediate understanding, they can situate the patients into a narrative that tells them what actions and reasons to expect and why to expect them.

Even more important than the starting problem, IT is able to overcome both the diversity problem and the geocentric bias. As already explained, ST can only accept the diversity problem as a necessary flaw in our ability to understand others. However, as Gallagher said, we are at least sometimes able to understand others that are very different from us, such as aliens and monsters (from movies), and people from different cultures (Gallagher 2012, p. 370). What allows us to do this, however, is not a simulation, but rather the knowledge we have of their stories. We only understand these aliens and foreigners when we are able to "frame their behavior in a narrative that informs us about their history or their situation" (Gallagher 2012, p. 370). We have diverse narratives that allow us to understand others in diverse contexts-ones that are not limited to the experiences that we have had in our individual lives (Gallagher 2012, p. 370). This also helps to show why we are better able to understand those who are close or similar to us, and reciprocally why it is more difficult to empathize with nonhuman animals and aliens. We share similar narratives to those close and similar to us-we "already know the general lines of their 
stories"-making it easier for me to see how they fit into that narrative (Gallagher 2012, p. 370).

The reason IT is able to overcome these problems is because it differs from ST in its most basic assumptions. To begin with, it does not start from the perspective that other minds are entirely inaccessible to me (Gallagher 2004, p. 204, 206, 2009, p. 292). Whereas ST argues in favor of an ability to understand other inaccessible minds, which cannot be perceived by us-trying to simulate and take the perspective of what those minds would be like-IT argues that "we directly perceive the other person's intentions, emotions, and dispositions in their embodied behavior" (Gallagher 2009, p. 292). This is what is explained by primary and secondary intersubjectivity. We already understand others intentions and desires in a very basic way (Gallagher 2004, pp. 208-209).

Additionally, unlike ST, IT maintains our context of being with others, rather than separating ourselves as observers (Gallagher 2004, pp. 207-208, 2009, p. 292). In this way, it resists "medical paternalism" in a way that ST did not (Hooker 2015, p. 546). For ST, I separate myself from the other in order to understand the other. I turn the other into an object of study, so that I can approach the other scientifically. IT argues that we do not normally understand others in third-person observation, but rather in "second-person interaction" (Gallagher 2009, p. 292). We are always interacting with others in some context, and it is in these interactions that we come to understand others. IT allows the patient to tell his or her story, which can prove both relaxing for the physicians (Halpern 2014, p. 309), and empowering for the patient (Halpern 2014, p. 303).

Though we should be careful here, since some may want to argue that narrative empathy does not actually overcome the problem of paternalism. The power relationships that this empathy was supposed to overcome instead "are rendered omnipresent, subtle and productive of new forms of selfhood and of patient-doctor relations" (Hooker 2015, p. 546). There is still the risk that the physician will be taking a pastoral position with regard to the patient (Mayes 2009). The patient is seen as a "confessing subject and requires that the doctor elicit, listen to, and ultimately interpret, the patient's "story," as if the patient was giving a confession (Hooker 2015, pp. 546-547). However, this is not necessarily the case, since IT is not a one-sided experience and judgment of the patient. In the establishment of the narrative, patient and physician can communicate back and forth, growing and focusing the narrative together (Ratcliffe 2014, p. 276). In this way, empathy remains a more other-directed practice (Ratcliffe 2014, p. 271).

To summarize, there are many benefits to adopting IT. It provides an explanation of empathy that matches and actually explains the way that empathy allows physicians to understand patients. Primary and secondary intersubjectivity provide a very basic understanding of others and narrative competency provides a more complex understanding of others. Additionally, possessing narratives allows for others to be immediately situated into narratives, allowing for initial understanding. This overcomes the starting problem and creates a foundation on which further interaction can build narratives and understanding. Furthermore, these levels of IT allow physicians to understand patients from different backgrounds and in various situations. Narratives allow others to be part of the understanding, making the understanding more other-focused and capable of being more diverse than the subjects narratives alone. Again, if better patient care is directly connected to how well physicians understand their patients, then IT is a better theory of empathy for medicine because it explains how this understanding is achieved.

\section{The relation between simulations and narratives}

While IT and similar approaches have started to gain some support, both philosophically and in the medical field (Halpern 2003, 2014; Ratcliffe 2014), it can still be asked whether or not IT is the best theory of medical empathy. Some have noted that approaches such as these can "run the risk of sounding romantic or lacking in rigour" (Hooker 543). The reason for this being that the first two levels of intersubjectivity argue that we have a basic understanding of others well before the application of theories or simulations, but they do not explain how this understanding happens. In other words, the argument presented is a negative one-that ST cannot explain this basic understanding, but that this basic understanding does exist. However, the possibility that ST and IT could work well together should be considered. It might be that ST provides the explanation for primary intersubjectivity that is needed.

This would not work if the simulation needs to be an explicit process - a conscious taking of the other's perspective. As explained in an earlier section, this is because infants are capable of understanding very basic similarities about self and other, but are not capable of running a highly cognitive process like a simulation. On the other hand, ST might be able to overcome the problem of infant simulation by appealing to mirror neuron or some other neurological research (Brothers 1989, p. 13; Iacoboni 2009; Iacoboni and Dapretto 2006; Chakrabarti and Baron-Cohen 2006; Preston and De Waal 2002; Gallese 2003). Mirror neurons are special neurons that people (as well as several other animals) possess that regularly fire both when I perform an action and when I observe others performing a similar action. For instance, my mirror neurons will fire when I reach for my mug to take a sip of coffee, as well as when I watch my friend reach for a mug to take a sip of coffee. The intention of the action is important here. The neurons 
won't fire if I simply observe my friend's hand on a desk. They will only fire if there is an intentional action that is similar to my own (Iacoboni 2009, pp. 75-77). This has been taken to be good support for the existence of immediate simulations taking place below the level of consciousness. As far as physicians are concerned, this would mean that they empathize with their patients so far as their mirror neurons fire, matching the intention of their patient's actions. Furthermore, infants can have mirror neurons from birth, allowing them to subconsciously simulate and understand the expressions of others.

On the other hand, I am hesitant to say that mirror neurons support ST, and by extension that ST, can fit the role of explaining primary intersubjectivity. For one, mirror neuron research is still very much in its adolescence and it is hard to say whether or not it has the implications for empathy (as well as a number of other theories) that some predict. The results of mirror neuron research as it applies to simulation theory is contentious (Turner 2012). Therefore, caution should be taken with regard to this research for the time being. Furthermore, there may be less similarity between the firing of neurons and mirror neurons than it is initially made to seem (Gallagher and Zahavi 2012, pp. 200-201). ${ }^{12}$ There is some correlation, but it might not be enough to say that it is a sufficient mirroring of the other's mental states. Therefore, even though ST may be able to fulfill the role of basic and immediate understanding of the other, it cannot do so based on conscious simulation, and there is also not enough support for it to do so via unconscious simulation. Perhaps when more research is done, a better answer will be able to be given.

The other option, which seems more likely, is that simulations are based on IT, and specifically narrative competency. There is no denying that we sometimes make use of simulates, specifically when we do not have the ability to communicate with others to construct narratives. Since even implicit simulation is not able to overcome the starting problem, any simulations of the other would already need to be based on some level of narrative competency. That is, out imaginings of other's situations must already be grounded in narratives (Gallagher 2012, p. 370). In this way, simulations can be seen as useful in some situations, but only so far as we simulate being in narratives that we have already constructed.

\section{The problem of time constraints}

While there may be objections that can be raised against IT, as with all theories, there is at least one that is directly relevant to the medical field and therefore warrants being

\footnotetext{
12 They also note several other problems with internal, unconscious simulation in this work.
}

addressed here. Halpern fairly summarizes the objection in the following way:

How practical is it to expect empathic communication given the demands of medical practice todayexcessive work load, tremendous time pressure, unfamiliar patients, and duties that vary from comforting to performing invasive procedures? What is practical, and often appropriate, is limited or partial empathic engagement. (Halpern 2014, p. 304)

While she is not talking directly about IT, the objection applies equally well to the construction of narrative, since they require communication. The worry is that IT is not a practical theory of empathy to endorse, since physicians do not have the amount of time available to construct narratives for every one of their patients. If true, this would be very problematic for IT, since it would not actually fit well with the requirements of a physician. However, three things can be said against this argument.

First, it can be argued that the physician's actual lack of time does not necessarily mean that they should not spend more time and establish narratives. In other words, it is true that the current structure of medical interactions does not allow for the proper amount of time to establish narratives, but this may be more of a problem with the expectation of the medical interaction than the theory of empathy. It may be that the structure of the medical interaction needs to be restructured to allow for empathy. As Halpern argues, "the current time and effort demands of practice can be dehumanizing for physicians, and therefore for patients as well" (Halpern 2014, p. 310). Proper empathetic interaction requires more time than is currently allowed. However, that the current structure of medical interactions does not allow for the best amount of time to be spent on physician-patient interactions does not mean that this objection can be dismissed. It may be a real problem for IT that time is limited.

A second argument is that empathy for IT does not always require one to go through all the levels of empathy. It is often the case that understanding a patient's situation and prescribing a treatment can be achieved at the levels of primary and secondary intersubjectivity, which are automatic and less time-consuming. These levels of empathy allow for an "immediate appreciation of someone's experience, rather than a two-step process involving simulation and inference" (Ratcliffe 2014, p. 271). This feature of IT is especially important for medicine because "in many contexts it is simply unnecessary for clinicians to engage in any kind of empathy beyond acknowledging the patient as a human being with feelings and worth" (Halpern 2014, p. 305). This is one of the strengths of IT, in that it allows for different levels of empathic engagement depending on the situation. Empathy should not be viewed as one overarching skill that must be achieved in every interactions 
(Halpern 2014, p. 304). It is a multileveled skill that can be accomplished more or less in depth. Therefore, teaching medical students how to empathize will involve teaching them to understand and implement different ways of empathizing (Halpern 2014, p. 304).

Third, it can be argued that establishing a narrative is not as time consuming as it may initially seem. The worry is that physicians will need to sit down and have long conversations with patients in order to develop a sufficient narrative to warrant an understanding. However, this is not the case. It has been shown that allowing the patient to establish a narrative by explaining his or her situation takes approximately $2 \mathrm{~min}$ for $80 \%$ of those patients (Langewitz et al. 2002). There are some that take more or less time, but 2 min is a short amount of time to establish an empathic understanding of the patient. Additionally, the narrative does not need to be a complete narrative of the entire person's life, which would be far too time consuming. Rather, physicians would ask specific questions to allow patients to fill in the information the physician already has based on their previous narratives, such as "Is there anything else about your situation you think I should know about?" or "Are there any thoughts and/or feeling you have that I might be missing?" This allows for the most complete and relevant narrative, in the shortest amount of time.

\section{Conclusion}

To summarize, there is currently a problematic lack of empathy in both medical students and physicians. The cause for this lack of empathy can be attributed to a number of factors, including the copious amount of information to be learned, the distrust of affective practices, the fear of risky personal connections, and the need for a coping strategy to avoid professional burnout. The result is that empathy is seen as a problem for the prevailing scientific attitude in medicine-it is a dangerous and extraneous experience that ought to be avoided. However, the singular importance of the scientific attitude as it has been appropriated for medicine is now being questioned, and empathy and other affective practices are seen as additionally important. Empathy is beneficial, leading to a greater amount of shared information, perceive care, trust, and empowerment for the patient, as well as a greater sense of fulfillment and lower chance of professional burnout for physicians. The goal then was to overcome the scientific attitude and revalue empathy within medicine (which itself should also help to overcome the attitude).

While ST is a popular explanation for how empathy allows us to understand others, there are a number of objection and limitations that make it less than ideal for medicine. As the diversity problem and the geocentric bias demonstrate, ST is limited in the way that it allows physicians to understand their patients. That is, physicians are limited in what they can imagine of the patient's situation, leaving little diversity beyond the first-person perspective of the physician. Additionally, ST is a theory of empathy that appropriates empathy into the scientific attitude, perpetuating rather than solving the problems explained in the first section. It is a theory of empathy that is still based on observation and judgment in order to understand the other.

IT, on the other hand, serves as a good theoretical basis for understanding how physicians empathize at different levels with different patients. When it comes to interacting with patients, physicians have a basic understanding of their thoughts and intentions, as provided by primary and secondary intersubjectivity. When needed, physicians can ask additional questions to allow the patient to expand his or her narrative. As such, IT overcomes the diversity problem and geocentric bias by making the physician's understanding of the patient more of a second-person interactions than a third-person observation. This refocusing shifts away from the scientific attitude and focuses more on communication and interaction. It is these skills that are more likely to lead to a better understanding of the patient, as well as the benefits listed above. This construction of narratives causes greater communication, which leads to better understanding, improved diagnostic accuracy, increased feelings of care, and increased trust.

As such, IT is the theory of empathy that best fits good medical practice, and is therefore the kind of empathy that should be encouraged in physicians. When found lacking, narrative competency - including the ability to ask relevant questions to quickly establish the most relevant narratives - is the skill that needs to be improved to improve empathy. This is not meant to imply that IT is the only ability that the physician needs to provide the best medical care. Other affective abilities, such as sympathy and care, also need to be fostered, but IT is the theory of empathy that ought to accompany these other abilities.

\section{References}

Batson, C.Daniel. 2009. These things called empathy: eight related but distinct phenomena. In The social neuroscience of empathy, ed. Jean Decety, and William Ickes. Cambridge: MIT Press.

Brothers, Leslie. 1989. A biological perspective on empathy. American Journal of Psychiatry 146(1): 10-19.

Chakrabarti, Bhismadev, and Simon Baron-Cohen. 2006. Empathizing: neurocognitive developmental mechanisms and individual differences. Progress in Brain Research 156: 403-417.

Cohen, Milton, et al. 2011. Stigmatization of patients with chronic pain: the extinction of empathy. Pain Medicine 12: 1637-1643. 
De Vignemont, Frédérique, and Pierre Jacob. 2012. What is it like to feel another's pain? Philosophy of Science 79(2): 295-316.

Derksen, Frans, Jozien Bensing, and Antoine Lagro-Janssen. 2013. Effectiveness of empathy in general practice: a systematic review. British Journal of General Practice 63(606): e76e84.

Di Blasi, Zelda, and Jos Kleijnen. 2003. Context effects: powerful therapies or methodological bias? Evaluation and the Health Professions 26: 166-179.

Eikeland, Hanne-Lise, et al. 2014. The physician's role and empathy - a qualitative study of third year medical students. BMC Medical Education 14: 165-172.

Finset, Arnstein, and Trond Arne Mjaaland. 2009. The medical consultation viewed as a value chain: a neurobehavioral approach to emotion regulation in doctor-patient interaction. Patient Education and Counseling 74(3): 323-330.

Gallagher, Shaun. 2004. Understanding interpersonal problems in autism: interaction theory as an alternative to theory of mind. Philosophy, Psychiatry, \& Psychology 11(3): 199-217.

Gallagher, Shaun. 2009. Two problems of intersubjectivity. Journal of Consciousness Studies 16(6-8): 289-308.

Gallagher, Shaun. 2012. Empathy, simulation, and narrative. Science in Context 25(3): 355-381.

Gallagher, Shaun, and Andrew N. Meltzoff. 1996. The earliest sense of self and others. Philosophical Psychology 9: 213-236.

Gallagher, Shaun, and Dan Zahavi. 2012. The phenomenological mind, 2nd ed. New York: Routledge.

Gallese, Vittorio. 2003. The roots of empathy: the shared manifold hypothesis and the neural basis of intersubjectivity. Psychopathology 36(4): 171-180.

Garden, Rebecca. 2007. The problem of empathy: medicine and the humanities. New Literary History 38(3): 551-567.

Gelhaus, Petra. 2012a. The desired moral attitude of the physician: (I) empathy. Medicine, Health Care and Philosophy 15: 103-113.

Gelhaus, Petra. 2012b. The desired moral attitude of the physician: (II) compassion. Medicine, Health Care and Philosophy 15: $397-410$

Gelhaus, Petra. 2013. The desired moral attitude of the physician: (III) care. Medicine, Health Care and Philosophy 16: 125-139.

Gerdes, Karen E. 2011. Empathy, sympathy, and pity: 21st-century definitions and implications for practice and research. Journal of Social Service Research 37: 230-241.

Gladstein, Gerald A. 1983. Understanding empathy: integrating counseling, developmental, and social psychology perspectives. Journal of Counseling Psychology 30(4): 467-482.

Gleichgerrcht, Ezequiel, and Jean Decety. 2013. Empathy in clinical practice: how individual dispositions, gender, and experience moderate empathic concern, burnout, and emotional distress in physicians. PLOS ONE 8(4): 1-12.

Halpern, Jodi. 2001. From detached concern to empathy: humanizing medical practice. New York: Oxford University Press.

Halpern, Jodi. 2003. What is clinical empathy? Journal of General and Internal Medicine 18: 670-674.

Halpern, Jodi. 2011. Clinical empathy. In From bench to bedside: Empathy and the social brain, ed. Decety Jean. Cambridge, MA: MIT Press.

Halpern, Jodi. 2014. From idealized clinical empathy to empathic communication in medical care. Medicine, Health Care and Philosophy 17: 301-311.

Hojat, Mohammadreza, et al. 2001. The Jefferson scale of physician empathy: development and preliminary psychometric data. Educational and Psychological Measurement 61(2): 349-365.

Hojat, Mohammadreza, et al. 2004. An empirical study of decline in empathy in medical school. Medical Education 38: 934-941.
Hojat, Mohammadreza, et al. 2009. The devil is in the third year: a longitudinal study of erosion of empathy in medical school. Academic Medicine 84(9): 1182-1191.

Hojat, Mohammadreza, et al. 2011. Physicians' empathy and clinical outcomes for diabetic patients. Academic Medicine 86: 359-364.

Hojat, Mohammadreza, et al. 2013. Empathy and health care quality. American Journal of Medical Quality: The Official Journal of the American College of Medical Quality 28(1): 6-7.

Hooker, Claire. 2015. Understanding empathy: why phenomenology and hermeneutics can help medical education and practice. Medicine, Health Care and Philosophy 18: 541-552.

Iacoboni, Marco. 2009. Mirroring people: the science of empathy and how we connect with others. New York: Picador.

Iacoboni, Marco, and Mirella Dapretto. 2006. The mirror neuron system and the consequences of its dysfunction. Nature Reviews Neuroscience 7(12): 942-951.

Khanuja, S., et al. 2011. Empathy and sympathy in the medical profession: should we stop the desertion? Pravara Medical Review 3(3): 37-39.

Kim, Sung Soo, Stan Kaplowitz, and Mark V. Johnston. 2004. The effects of physician empathy on patient satisfaction and compliance. Evaluation and the Health Professions 27: 237-251.

Langewitz, Wolf, et al. 2002. Spontaneous talking time at start of consultation in outpatient clinic: cohort study. British Medical Journal 325: 682-683.

Lanzoni, Susan. 2012. Empathy in translation: movement and image in the psychological laboratory. Science in Context 25(3): 301-327.

Larson, Eric B., and Xin Yao. 2005. Clinical empathy as emotional labour in the patient-physician relationship. JAMA 293(9): $1100-1106$.

Macnaughton, Jane. 2009. The dangerous practice of empathy. The Lancet 373: 1940-1941.

Marcus, E.R. 1999. Empathy, humanism and the professionalisation process of medical education. Academic Medicine 74: 1211-1215.

Mayes, Christopher. 2009. Pastoral power and the confessing subject in patient-centred communication. Bioethical Inquiry 6: 483-493.

Meltzoff, Andrew N., and M. Keith Moore. 1977. Imitation of facial and manual gestures by human neonates. Science 198: 75-78.

Pedersen, Reidar. 2008. Empathy: a wolf in sheep's clothing? Medicine, Health Care and Philosophy 11: 325-335.

Pedersen, Reidar. 2009. Empirical research on empathy in medicine-a critical review. Patient Education and Counseling 76(3): 307-322.

Pedersen, Reidar. 2010. Empathy development in medical education-a critical review. Medical Teacher 32: 593-600.

Preston, Stephanie D., and Frans B.M. de Waal. 2002. Empathy: its ultimate and proximate bases. Behavioral and Brain Sciences 25(1): $1-20$.

Ratcliffe, Matthew. 2014. The phenomenology of depression and the nature of empathy. Medicine, Health Care and Philosophy 17: 269-280.

Roter, Debra L., et al. 1998. Effectiveness of interventions to improve patient compliance: A meta-analysis. Medical Care 36(8): $1138-1161$

Rushton, John Philippe. 1980. Altruism, socialization, and society. Englewood Cliffs: Prentice-Hall.

Shanafelt, Tait D., et al. 2005. Relationship between increased personal well-being and enhanced empathy among internal medicine residents. Journal of General Internal Medicine 20: $559-564$.

Singh, Sonal. 2005. Empathy: lost or found in medical education? Medscape General Medicine 7(3): 74. 
Skeff, Kelley M., and Sunita Mutha. 1998. Role models: guiding the future of medicine. New England Journal of Medicine 339: 2015-2017.

Stein, Edith. 1989. On the Problem of Empathy. Third Revised. (trans: Waltraut Stein). Washington, DC: ICS Publications.

Suchman, Anthony, et al. 1997. A model of empathic communication in the medical interview. JAMA 277(8): 678-682.

Svenaeus, Fredrik. 2015. The relationship between empathy and sympathy in good health care. Medicine, Health Care and Philosophy 18: 267-277.

Tavakol, Sina, Reg Dennick, and Mohsen Tavakol. 2012. Medical students' understanding of empathy: a phenomenological study. Medical Education 46(3): 306-316.
Testerman, J.K., K.R. Morton, L.K. Loo, J.S. Worthley, and H.H. Lamberton. 1996. The natural history of cynicism in physicians. Academic Medicine 71(10): S43-S45.

Turner, Stephen. 2012. The strength of weak empathy. Science in Context 25(3): 383-399.

Ward, Julia, et al. 2012. The empathy enigma: an empirical study of decline in empathy among undergraduate nursing students. Journal of Professional Nursing 28(1): 34-40.

Williams, et al. 2015. Student empathy levels across 12 medical and health professions: an interventional study. Journal of Compassionate Health Care 2: 4-9.

Zahavi, Dan. 2014. Self and other. Oxford: Oxford University Press. 